\title{
RELAXATION OSCILLATIONS, PULSES, AND TRAVELLING WAVES IN THE DIFFUSIVE VOLTERRA DELAY-DIFFERENTIAL EQUATION*
}

\author{
LUIS L. BONILLA $\dagger$ AND AMABLE LIÑÁN $\ddagger$
}

\begin{abstract}
The diffusive Volterra equation with discrete or continuous delay is studied in the limit of long delays using matched asymptotic expansions. In the case of continuous delay, the procedure was explicitly carried out for general normalized kernels of the form $\sum_{n=p}^{N} g_{n}\left(t^{n} / T^{n+1}\right) e^{-t / T}, p \geqq 2$, in the limit in which the strength of the delayed regulation is much greater than that of the instantaneous one, and also for $g_{n}=\delta_{n 2}$ and any strength ratio.

Solutions include homogeneous relaxation oscillations and travelling waves such as pulses, periodic wavetrains, pacemakers and leading centers, so that the diffusive Volterra equation presents the main features of excitable media.
\end{abstract}

1. Introduction. The aim of the present work is to study the solutions of the diffusive Volterra delay-differential equation for long time lags.

The following equation was proposed in the thirties by Volterra to describe the evolution in the laboratory of small organism species with short generation times:

$$
\begin{aligned}
& d N / d t=r N\left\{1-N / K-Q^{-1} \int_{-\infty}^{t} N(\tau) G(t-\tau) d \tau\right\} \quad(t>0), \\
& N(t)=\Phi(t) \geqq 0 \quad(t \leqq 0), \\
& \int_{0}^{\infty} G(\tau) d \tau=1, \quad G(\tau)>0 \quad \text { for } \tau>0, \\
& r, Q, K \text { are }>0 .
\end{aligned}
$$

Here, $N$ is the population density of the species, whose birth rate is $r, K$ is the carrying capacity of the environment in the absence of delay and the integral term accounts for environmental pollution due to waste products and dead organisms [13].

We shall consider weight functions $G(t)$ that decay exponentially like $t^{r} e^{-t / T}$ as $t \rightarrow \infty(r>0)$, so that it is possible to write

$$
G(t)=\sum_{n=0}^{N} g_{n} G^{(n)}(t)
$$

for an appropriate integer $N$. Here,

$$
G^{(n)}(t)=\frac{t^{n}}{T^{n+1}} e^{-t / T}
$$

and therefore,

$$
\int_{0}^{\infty} G^{(n)}(t) d t=1, \quad \sum_{n=0}^{N} g_{n}=1
$$

* Received by the editors August 5, 1982, and in revised form March 8, 1983.

$\uparrow$ Department of Mathematics, Stanford University, Stanford, California 94305, and Departamento de Física Fundamental, UNED, Apartado de Correos 50487, Madrid, Spain. The research of this author was supported by the Office of Naval Research, the National Science Foundation, the Air Force Office of Scientific Research, the Army Research Office and by the U.S.-Spain Joint Committee for Scientific and Technological Cooperation.

‡E.T.S. Ing. Aeronáuticos, Universidad Politécnica de Madrid, Madrid, Spain. 
In the limit of infinitely spiked kernels, (1.1) is reduced to the discrete form

$$
\frac{d N}{d t}=r N\{1-N / K-N(t-T) / Q\}
$$

Equations (1.1) or (1.8) can also describe the evolution of a herbivorous species population subject to the (instantaneous) action of a predator (the $-N / K$ term) and whose nutrient regenerates itself after a time $T$ (the delayed regulatory term) [15], [16], [19].

If we now assume that the movement of the species obeys the random diffusion hypothesis, the population density will evolve according to the following diffusive Volterra equation on an infinitely extended one-dimensional support (for the sake of the simplicity) [17]:

$$
\frac{\partial N}{\partial t}=D \frac{\partial^{2} N}{\partial x^{2}}+r N\left\{1-N / K-Q^{-1} \int_{0}^{\infty} G(\tau) N(x, t-\tau) d \tau\right\}
$$

where, if we put $G(\tau)=\delta(\tau-T)$, we recover the discrete delay $N(x, t-T)$ as in (1.8).

In the absence of delays, (1.9) has an unstable homogeneous steady solution, $N=0$, the extinction state, and a stable one $N_{0}=K Q /(K+Q)$. The main effect of the time lag, $T$, is to destabilize the homogeneous steady state $N_{0}$ in (1.9), thereby generating a time periodic solution of increasing amplitude as $T$ increases. The various dissipative structures that can be found near the bifurcation point were described in a number of papers [1], [2], [12], [21]. In this paper, we shall study the solutions of (1.1)-(1.9) in the limit of very long delays using matched asymptotic expansions.

Defining the following dimensionless parameters and variables in (1.9):

$$
\begin{aligned}
& \tilde{x}=x(D T)^{-1 / 2}, \quad \tilde{t}=t / T, \quad u=N / K, \\
& \alpha=r T, \quad \beta=Q / K, \quad \tilde{G}(\tilde{t})=T \cdot G(t)
\end{aligned}
$$

gives the resultant dimensionless equation

$$
\frac{\partial u}{\partial t}=\frac{\partial^{2} u}{\partial x^{2}}+\alpha u\left\{1-u-\beta^{-1} \int_{0}^{\infty} G(\tau) u(x, t-\tau) d \tau\right\}
$$

in which we have suppressed the tildes to simplify the writing.

Near the bifurcation point, $\alpha=\alpha_{0}$, the smallness of the parameter $\varepsilon \sim\left|\alpha-\alpha_{0}\right|^{1 / 2}$ can be exploited to study the bifurcating time-periodic solutions of (1.11) by means of the method of multiple scales [1], [2], [12], [21]. On the other hand, if the dimensionless delay $\alpha$ in (1.11) is very large $(\alpha \rightarrow \infty)$, as it shall be assumed in this paper, the time periodic solutions of (1.11) can again be described by asymptotic methods, in this case, by the method of matched asymptotic expansions. The asymptotically stable solutions of (1.11) without diffusion are either the steady state $u_{e}=\beta /(1+\beta)$ or a relaxation-like limit cycle, depending on the value of $\beta$. If the diffusion is included, we can have periodic wave-trains and pulses depending on the initial condition.

The rest of this paper is organized as follows.

In $\S 2$, we shall study transient stages and homogeneous limit cycles when the delay in the regulatory term of (1.11) is of the simple form $G(\tau)=\tau^{2} e^{-\tau} / 2\left(g_{n}=\delta_{n 2}\right.$ in (1.5)).

In $\S 3$, we will consider the effect of the diffusion on the above described situation. New solutions include travelling waves that advance with the velocity of the wavefront solutions of the Fisher equation. The general kernel (1.5) will be considered in $\S 4$ where explicit results will be obtained in the limit $\beta \rightarrow 0$. 
In $\S 5$, the case of discrete delays $(G(\tau)=\delta(\tau-1)$, the Dirac delta function), with or without diffusion, is studied, and, finally, a discussion of our results and a comparison with other results in the literature constitutes $\S 6$.

2. Homogeneous relaxation-like limit cycles and transitory stages for continuous delay $g_{n}=\delta_{n 2}$. The steady solution $u_{e}=\beta /(1+\beta)$ of the homogeneous Volterra equation

$$
\frac{d u}{d t}=\alpha u\left\{1-u-\beta^{-1} \int_{0}^{\infty} G(\tau) u(t-\tau) d \tau\right\}
$$

is, in the case $g_{n}=\delta_{n p}$, asymptotically stable (A.S.) for large values of $\alpha$, if $\beta>\beta_{0}$ with

$$
\beta_{0} \equiv \cos ^{p+1}(\pi /(p+1)), \quad p=0,1, \cdots,
$$

and unstable if $0 \leqq \beta \leqq \beta_{0}$. For $p=0,1$, the solution $u_{e}$ is A.S. for all positive values of $\beta$, if $\alpha \gg 1$. Therefore, only if $p \geqq 2$ is it possible to get an unstable $u_{e}$ for $\alpha \rightarrow \infty$. As the qualitative behavior of (2.1) should be the same for any $p \geqq 2$, we shall study the simpler case $p=2$. In this case, the steady solution is unstable if $0 \leqq \beta<\frac{1}{8}$ and A.S. if $\frac{1}{8}<\beta$, for $\alpha \gg 1$.

In the general case, the characteristic equation which determines the linear stability of $u_{e}$ can be solved easily in the limit $\beta^{1 /(p+1)} \ll 1, p$ being the smallest integer such that $g_{n} \neq 0$; then $\left(g_{p} / \beta\right)^{1 /(p+1)} \cos (\pi /(p+1))$ is the largest real part of the characteristic roots, and again only for $p \geqq 2$ it is possible to have an unstable $u_{e}$. We shall go back to the limit $\beta^{1 /(p+1)} \rightarrow 0$ in the general case in $\$ 4$, where the procedure here illustrated for $g_{n}=\delta_{n 2}$ will be extended.

Using the Volterra change of variables (linear chain trick) [13]

$$
\begin{gathered}
v=\int_{0}^{\infty} G^{(0)}(\tau) u(t-\tau) d \tau, \\
w=\int_{0}^{\infty} G^{(1)}(\tau) u(t-\tau) d \tau, \\
z=\int_{0}^{\infty} G^{(2)}(\tau) u(t-\tau) d \tau,
\end{gathered}
$$

(2.1) can be put in the form

$$
\frac{d u}{d t}=\alpha u(1-u-z / \beta)
$$

The change (2.3) transforms the initial condition of (2.1),

$$
u(\tau)=\phi(\tau), \quad-\infty \leqq \tau \leqq t_{0},
$$

into the following initial condition for (2.4),

$$
u\left(t_{0}\right)=\phi\left(t_{0}\right), \quad v\left(t_{0}\right)=\int_{0}^{\infty} G^{(0)}(\tau) \phi\left(t_{0}-\tau\right) d \tau, \quad \text { etc. }
$$

Let us suppose that the initial condition (2.6) is very close to the extinction state $u=v=w=z=0$; for example, $u\left(t_{0}\right)=a_{0} / \alpha, v\left(t_{0}\right)=a_{1} / \alpha, w\left(t_{0}\right)=a_{2} / \alpha, z\left(t_{0}\right)=a_{3} / \alpha$, with $a_{i}$ of the order of unity. As the zero solution is unstable, in a first stage of duration 
$O(1 / \alpha), u(t)$ quickly increases to $u=1$ while $v, w, z$ remain $O(1 / \alpha)$. In this stage, the solution of (2.4) and (2.6) takes the form

$$
\begin{aligned}
u(t) & =u_{0}(\sigma)+\alpha^{-1} u_{1}(\sigma)+O\left(\alpha^{-2}\right), \quad \sigma=\alpha\left(t-t_{0}\right), \\
v(t) & =\alpha^{-1} v_{1}(\sigma)+O\left(\alpha^{-2}\right) \\
w(t) & =\alpha^{-1} w_{1}(\sigma)+O\left(\alpha^{-2}\right), \\
z(t) & =\alpha^{-1} z_{1}(\sigma)+O\left(\alpha^{-2}\right)
\end{aligned}
$$

Inserting (2.7) into (2.4), we obtain as a first approximation,

$$
\frac{d u_{0}}{d \sigma}=u_{0}\left(1-u_{0}\right)
$$

$$
\frac{d v_{1}}{d \sigma}=u_{0}, \quad \frac{d w_{1}}{d \sigma}=0, \quad d z_{1} / d \sigma=0
$$

The solution of $(2.8)$ is

$$
u_{0}=e^{\sigma} /\left(1+e^{\sigma}\right), \quad v_{1}=a_{1}+\ln \left(1+e^{\sigma}\right), \quad w_{1}=a_{2}, \quad z_{1}=a_{3},
$$

provided that the origin of time is chosen so that

$$
t_{0}=\alpha^{-1} \ln \left(a_{0} / \alpha\right) \quad\left(t_{0} \rightarrow 0^{-} \text {as } \alpha \rightarrow \infty\right) .
$$

When $\sigma \sim \alpha, u_{0}$ and $v, w$ and $z$ become $O(1)$, we therefore have a new stage described by the independent variable $t$, with $0<t \sim 1$, in which the solution of (2.1) is described by the following expansion,

$$
\begin{aligned}
& u=u_{0}^{1}(t)+\alpha^{-1} u_{1}^{1}(t)+O\left(\alpha^{-2}\right), \\
& v=v_{0}^{1}(t)+\alpha^{-1} v_{1}^{1}(t)+O\left(\alpha^{-2}\right), \quad \text { etc. }
\end{aligned}
$$

Hence, as long as $\alpha u$ remains $\gg 1$, the first approximation obeys the system

$$
\begin{aligned}
& 1-u-z / \beta=0, \\
& \frac{d v}{d t}=u-v, \quad \frac{d w}{d t}=v-w, \quad \frac{d z}{d t}=w-z,
\end{aligned}
$$

where we have suppressed sub- and superindices in order not to complicate the writing of the equations. The initial conditions for (2.11)

$$
u-1=v=w=z=0 \quad \text { at } t=0
$$

are obtained from the matching conditions with the previous stage.

The solution of (2.11)-(2.12) is a good approximation to the true solution up to terms of order $1 / \alpha$ so long as $z$ is smaller than $\beta$. System $(2.11 \mathrm{a}-\mathrm{d})$ is a linear system with constant coefficients, and thus can be solved exactly. This solution shows that if $\beta>\beta_{c 1}=0.383, z$ is always less than $\beta$ and that $u$ approaches $u_{e}$ in an oscillatory fashion as $t \rightarrow \infty$.

If $\beta<\beta_{c 1}$ the solution of (2.11)-(2.12) takes the value $u=0, v=v_{1}, w=w_{1}, z=\beta$ when $t=t_{1}(\beta)$. Clearly, as $t \rightarrow t_{1}(\beta), u$ becomes small so $\alpha u \gg 1$ is no longer true. After $t=t_{1}(\beta), u \ll 1$ so that we can approximate $(2.4 \mathrm{a})$ by $d u / d t=\alpha u(1-z / \beta)$. This leads to

$$
u / C=\exp \left\{\alpha \int_{t_{1}}^{t}(1-z / \beta) d t\right\}
$$




$$
\frac{d v}{d t}=-v, \quad \frac{d w}{d t}=v-w, \quad \frac{d z}{d t}=w-z
$$

The constant $C=C_{1} \alpha^{-1 / 2}$ can be evaluated by introducing an intermediate stage between (2.11)-(2.12) and (2.13), which is described by the equation

$$
\frac{d u}{d t}=-\alpha u\left\{u+\left(w_{1} / \beta-1\right)\left(t-t_{1}\right)\right\} .
$$

At this stage, $u(t)=\hat{u}(\tau) \alpha^{-1 / 2}, v(t)=\hat{v}(\tau), w(t)=\hat{w}(\tau), z(t)=\beta+\hat{z}(\tau) \alpha^{-1 / 2}, \quad \tau=$ $\alpha^{1 / 2}\left(t-t_{1}\right)$, and after the scaling $U=\left(w_{1} / \beta-1\right)^{-1 / 2} \hat{u}, \theta=\left(w_{1} / \beta-1\right)^{1 / 2} \tau$, we may write (2.14) in the following parameter-free form,

$$
\frac{d U}{d \theta}=-U(U+\theta)
$$

that must be solved with the initial condition

$$
\lim _{\theta \rightarrow-\infty} U / \theta=-1,
$$

insuring that the solution of $(2.15)-(2.16)$ matches the solution of $(2.11)-(2.12)$. The solution of $(2.15)-(2.16)$ is

$$
U(\theta)=(2 / \pi)^{1 / 2} e^{-\theta^{2} / 2}\left\{1+\operatorname{erf}\left(\theta / 2^{1 / 2}\right)\right\}^{-1},
$$

and the constant $C_{1}$ in $(2.13 \mathrm{a})$ is therefore

$$
C_{1}\left(w_{1} / \beta-1\right)^{-1 / 2}=C_{2}=\lim _{\theta \rightarrow \infty} e^{\theta^{2 / 2}} U(\theta)=(2 \pi)^{-1 / 2} .
$$

According to (2.13), $u$ again takes the value $C$ at $t=t_{2}$ given by

$$
\int_{t_{1}}^{t_{2}}(1-z / \beta) d t=0
$$

in which

$$
v\left(t_{2}\right)=v_{2}, \quad w\left(t_{2}\right)=w_{2}, \quad z\left(t_{2}\right)=z_{2}<\beta
$$

After $t=t_{2}$, the exponent in (2.13a) becomes positive and $u$ increases quickly, in a time $t-t_{2}=O(1 / \alpha)$, up to the value $\left(1-z_{2} / \beta\right)$ during a stage described by the equations

$$
\frac{d u}{d t}=\alpha u\left(1-u-z_{2} / \beta\right), \quad v=v_{2}, \quad w=w_{2}, \quad z=z_{2},
$$

so that

$$
u /\left(1-z_{2} / \beta\right)=e^{\sigma} /\left(1+e^{\sigma}\right)
$$

with

$$
\sigma=\alpha\left(1-z_{2} / \beta\right)\left(t-t_{2}\right)+\ln \left\{C_{1} \alpha^{-1 / 2}\left(1-z_{2} / \beta-C_{1} \alpha^{-1 / 2}\right)^{-1}\right\} .
$$

After this fast increment of $u$ around $t=t_{2}$, comes a new stage, of duration $O(1)$ in $t$, in which the evolution of $u$, as long as $u$ is positive, is again described by (2.11) with the initial conditions $(2.18 \mathrm{~b}-\mathrm{d})$. There is another value of $\beta, \beta_{\mathrm{c} 2}<\beta_{\mathrm{c} 1}$, such that when $\beta_{c 2}<\beta<\beta_{c 1}, u$ decays to $u_{e}$ in an oscillatory manner, without vanishing, as $t \rightarrow \infty$; whereas, if $\beta<\beta_{c 2}$, there is another time interval $\left(t_{3}, t_{4}\right)$ in which $u$ behaves 
as in $\left(t_{1}, t_{2}\right)$. After $t_{4}, u$ again rises to a value $\left(1-z_{4} / \beta\right)$ and another cycles begins. If $\beta<\beta_{c}, \beta_{c}=0.154$, the solution becomes periodic after a sufficiently long time, and every period is formed by two intervals $I_{1}$ and $I_{2}$ in which the behavior of $u$ is very different. During $I_{1}, u=1-z / \beta$ decreases from a certain value $\left(1-z_{k} / \beta\right)$ to zero (up to $O(1 / \alpha)$ terms) obeying (2.11); whereas, during the interval $I_{2}$ given by $\int_{I_{2}}(1-z / \beta) d t=0, u$ is approximately zero.

As can be seen from our construction, the relaxation-like limit cycle analyzed so far is phase A.S. and coexists with the A.S. steady solution $u=u_{e}$ for $\beta_{0}=0.125<\beta<$ $\beta_{c}=0.154$, thus displaying hysteretic phenomena as $\beta$ is varied. At $\beta=0.125$, there is a Hopf bifurcation from $u_{e}$ which is analyzed in Appendix 1. The situation is described in Fig. 1 where the hysteresis cycle is explicitly shown.

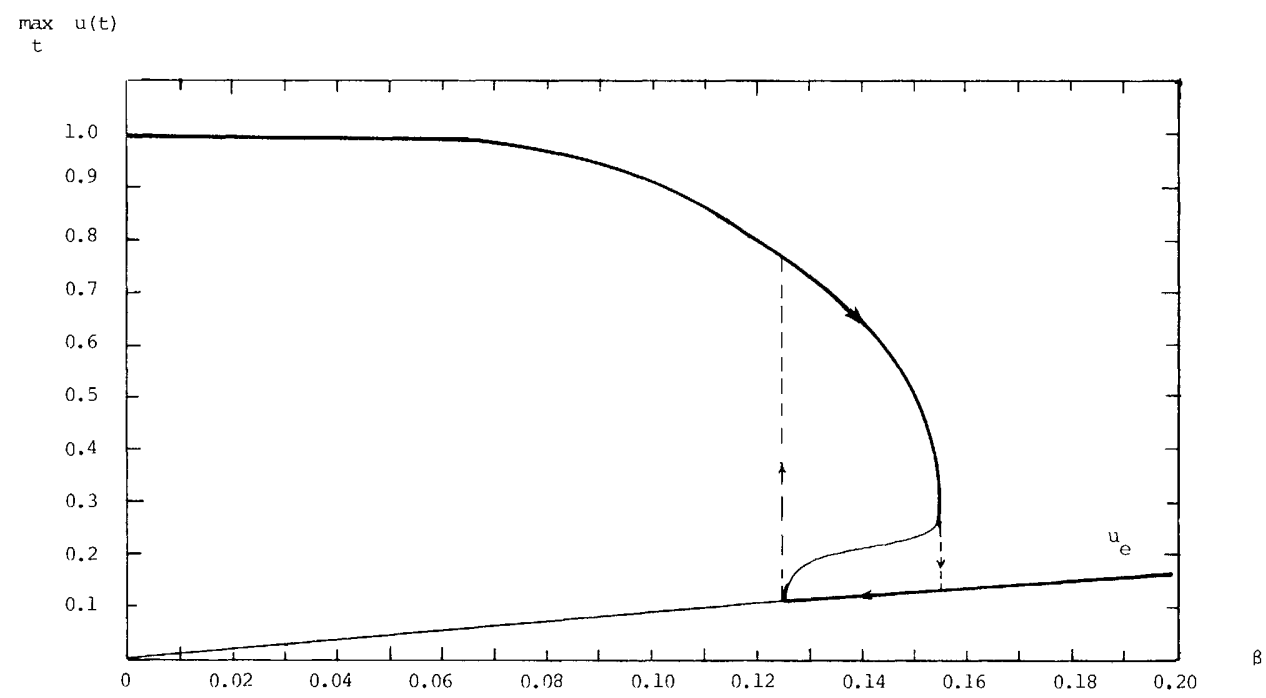

FIG. 1. Bifurcation diagram for the homogeneous Volterra equation with $g_{n}=\delta_{n 2}$. Heavy lines represent stable solutions whereas thin lines represent unstable solutions

Notice that in the asymptotic limit $\alpha \rightarrow \infty$, the solution can be described for $t>0$ by the system of linear equations (2.11), in the intervals when $u>0$, and by the linear equations $(2.13 \mathrm{~b}-\mathrm{d})$ supplemented by the following equation $(2.13 \mathrm{e})$ in the intervals where $u=0$, with the condition $I=0$ at the beginning and at the end of those intervals.

$$
\frac{d I}{d t}=1-z / \beta
$$

The solutions of the above mentioned linear equations can be easily written in explicit form, and the integrating constants associated with the different intervals can be determined by requiring continuity for all $t$ of the variables $v, w$ and $z$. As this procedure leads to transcendental equations for the duration of the time intervals (i.e., for the numbers $t_{1}, t_{2}$, etc.), which should be solved numerically, we have preferred to proceed stage by stage numerically integrating the linear equations (2.11) and $(2.13 \mathrm{~b}-\mathrm{e})$ alternately, together with the continuity requirement for $v, w$ and $z$.

The results of the asymptotic analysis that we have performed in this section are summarized in Fig. 2, where $u(t)$ is depicted, in the limiting case $\alpha \rightarrow \infty$, for two different values of $\beta$. For $\beta<\beta_{c}$, the asymptotic solution shows abrupt transitions involving jumps in $u$ or its time derivative, bounding intervals where $u=0$. 


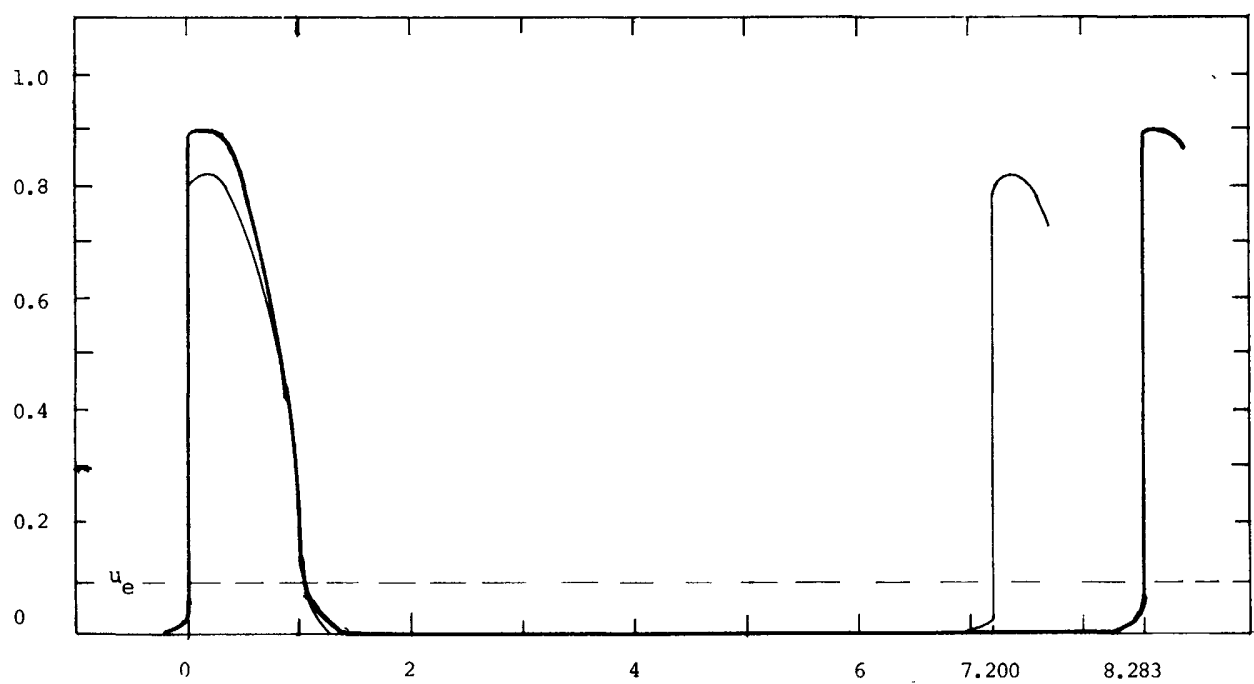

FIG. 2a. Periodic solution for $\beta=0.1$. After three pulses from $y=0$, the distance between successive maxima becomes equal to the period except for an error smaller than $0.1 \%$. An estimation of the period using the simple formula (see $\S 4): T=\eta_{1} \beta^{1 / 3}+W_{0}^{1} \beta^{-2 / 3}$ (homogeneous) or $T=\eta_{1} \beta^{1 / 3}+\left(8 W_{0}^{1}\right)^{1 / 2} \beta^{-1 / 3}$ (diffusive) gives us $T=7.25$ or $T=8.01$, respectively, which are of the same order as the true period depicted in the figure (error smaller than 13\%). Heavy lines correspond to relaxation oscillations and thin lines to travelling wavetrains.

u

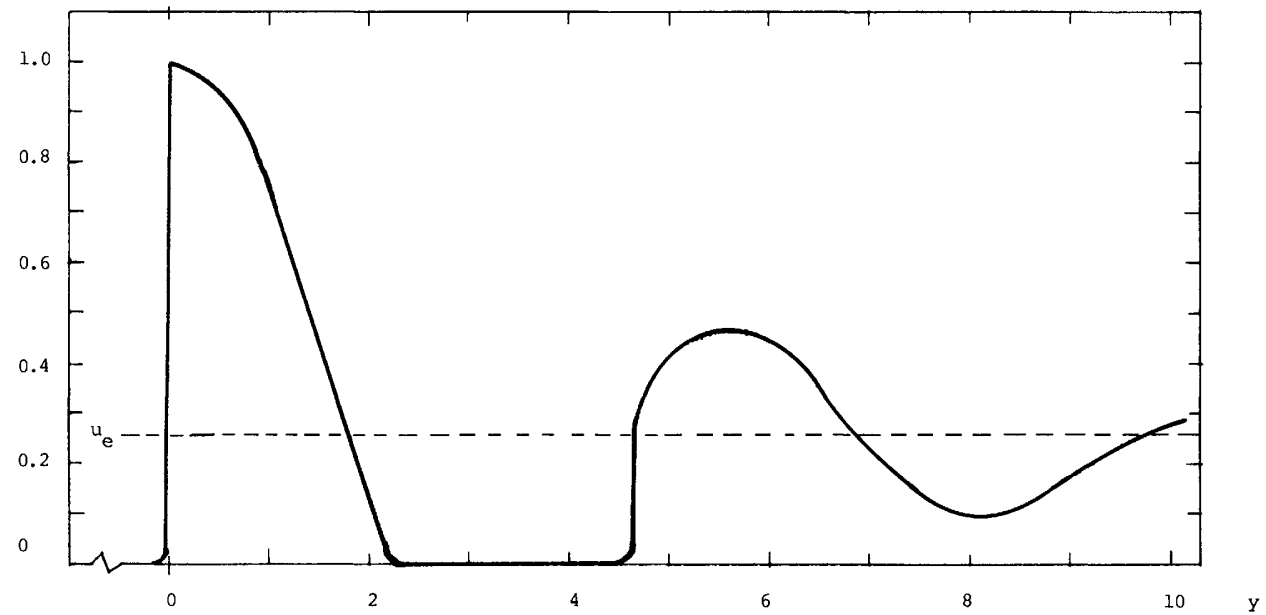

FIG. 2b. Solution for $\beta=0.3$. The steady state $u_{e}=0.23$ is A.S.

For $\beta<\beta_{c}$, u becomes periodic in $t$, with a sawtooth shape, after a transitory stage of similar shape.

3. The diffusive Volterra equation with continuous delay $\left(g_{n}=\delta_{n 2}\right)$. In this section, we shall study wave-like solutions of the following problem

$$
\frac{\partial u}{\partial t}=\frac{\partial^{2} u}{\partial x^{2}}+\alpha u\left\{1-u-\beta^{-1} \int_{0}^{\infty} G^{(2)}(\tau) u(x, t-\tau) d \tau\right\},
$$


or, equivalently, of the following system,

$$
\begin{aligned}
& \frac{\partial u}{\partial t}=\frac{\partial^{2} u}{\partial x^{2}}+\alpha u(1-u-z / \beta) \\
& \frac{\partial v}{\partial t}=u-v, \quad \frac{\partial w}{\partial t}=v-w, \quad \frac{\partial z}{\partial t}=w-z
\end{aligned}
$$

according to the Volterra change of variable (2.3).

We shall show that (3.1) or (3.2) has travelling wave solutions of the form $u(x, t)=u(y), y=t+x / c$, and will comment on the problem of the appropriate initial conditions later, in $\S 6$.

For travelling wave solutions, the system (3.2) can be written as

$$
\begin{aligned}
& \frac{d u}{d y}-c^{-2} \frac{d^{2} u}{d y^{2}}=\alpha u(1-u-z / \beta), \\
& \frac{d v}{d y}=u-v, \quad \frac{d w}{d y}=v-w, \quad \frac{d z}{d y}=w-z, \\
& y=t+x / c,
\end{aligned}
$$

and the boundary conditions that correspond are

$$
u=v=w=z=0 \quad \text { at } y \rightarrow-\infty,
$$

associated with the unstable steady state solution of (3.1), and the condition

$$
u=v=w=z=u_{e}=\beta /(1+\beta) \quad \text { at } y \rightarrow \infty
$$

associated with the stable steady state solution of (3.1), if $\beta$ is larger than a critical value $\beta_{c}$, to be determined later. For smaller values of $\beta$, the downstream boundary conditions $(3.4 \mathrm{~b})$ must be replaced by the condition that the solution should become periodic in $y$ for large positive values of $y$. In the first case the travelling wave solutions are pulses, while in the second case they lead to periodic wave trains.

The wavespeed $c$ is determined by the initial condition $u(x, t)$ at $t=0$. We shall discuss the initial value problem in $\S 6$. Here, we shall determine the asymptotic shape of the travelling waves and the admissible values of the speed $c$ (the travelling wave problem) for large values of $\alpha$.

For large $\alpha$, the structure of the travelling wave is similar to that corresponding to the homogeneous case. There is a first region of length $1 / \alpha$ in $y$ where $u$ rises from 0 to 1 , as described by the following boundary value problem

$$
\begin{aligned}
& \frac{d u}{d \eta}-\lambda \frac{d^{2} u}{d \eta^{2}}=u(1-u), \\
& u(-\infty)=0, \quad u(\infty)=1, \\
& \eta=\alpha y=\alpha(t+x / c), \\
& \lambda=\alpha / c^{2}
\end{aligned}
$$

while $v, w$ and $z$ are small, of order $\alpha^{-1}, \alpha^{-2}$, and $\alpha^{-3}$ respectively. Notice that the problem $(3.5 \mathrm{a}-\mathrm{d})$ corresponds to the initial-boundary value problem for the Fisher 
equation

$$
\begin{aligned}
& \frac{\partial u}{\partial \tau}=\alpha \frac{\partial^{2} u}{\partial \xi^{2}}+u(1-u), \quad u=u(\xi, \tau), \\
& u(-\infty, 0)=0, \quad u(\infty, 0)=1, \\
& \tau=\alpha t, \quad \xi=\alpha x .
\end{aligned}
$$

It is well known that (3.5) has a travelling wavefront solution that moves with asymptotic velocity $c=2 \alpha^{1 / 2}$ and that is an A.S. solution of (3.6). Actually there is a travelling wavefront solution of (3.5) that moves with velocity $c$ for each $\lambda=\alpha / c^{2}$, if $\lambda \varepsilon\left[0, \frac{1}{4}\right]$. The wavefront with speed $c$ is an asymptotically stable solution of (3.6) in the sense that if $u(\xi, 0)$ satisfies $(3.7 \mathrm{a}-\mathrm{c})$ below for some $c \geqq 2 \sqrt{\alpha}, u(x, t)$ evolves into the travelling wave with speed $c$ (except for a constant phase shift).

$$
\begin{aligned}
& u(\xi, 0)=a(\xi) \exp \{K(c) \xi\}[1+O(1)], \quad \xi \rightarrow-\infty, \\
& \alpha K(c)=c / 2-\left(c^{2} / 4-\alpha\right)^{1 / 2}, \\
& a(\xi)>0, \quad a\left(\xi+\xi_{0}\right) \sim a(\xi) \quad \text { as }|\xi| \rightarrow \infty,
\end{aligned}
$$

for constant $\xi_{0}$ [18]. If $u(\xi, 0)=0$ for $\xi<\xi_{c}$, the A.S. wavefront is the one with minimum velocity, $\lambda=\frac{1}{4}$, i.e., $c=2 \alpha^{1 / 2}[10]$.

From now on, we shall study only the case in which the solution of (3.5) is the Fisher wavefront that advances with asymptotic velocity $2 \alpha^{1 / 2}$. Wavefronts with larger velocities can be described in the same way and give rise to faster periodic wavetrains. In the limit $c \rightarrow \infty(\lambda \rightarrow 0)$, the homogeneous periodic solution of $\S 2$ is to be obtained.

The solution $u(\eta)$ of (3.5) ceases to be a good approximation to (3.3)-(3.4) when $\eta \gg 1$ so that $y=t+x / c=O(1)$; in this region $v, w$, and $z$ become or order 1 . For the description of this second region, we use $y$ as independent variable and rewrite (3.3a) in the form

$$
(\alpha u)^{-1} \frac{d u}{d y}-\left(4 \alpha^{2} u\right)^{-1} \frac{d^{2} u}{d y^{2}}=1-u-z / \beta .
$$

Equation (3.8) takes for $\alpha \rightarrow \infty$ the limiting form

$$
1-u-z / \beta=0,
$$

as long as $\alpha u \gg 1$. This equation together with (3.3b-d) and the initial conditions

$$
v=w=z=0 \quad \text { at } y=0,
$$

obtained from the matching conditions with the solution for the previous region, determine $u, v, w$ and $z$ as functions of $y$, in the first approximation for large $\alpha$. This approximation breaks down at $y=y_{1}$, such that $u\left(y_{1}\right)=0$. The system of equations $(3.3 \mathrm{~b}-\mathrm{d}),(3.9 \mathrm{a}-\mathrm{d})$, is exactly the system $(2.11)-(2.12)$ for the homogeneous Volterra equation, so that, as it was found in $\S 2$, the solution of $(3.3 \mathrm{~b}-\mathrm{d}),(3.9 \mathrm{a}-\mathrm{d})$ is an oscillatory decay toward the homogeneous steady solution $u_{e}$ for $y \rightarrow \infty$ if $\beta>\beta_{c_{1}}=$ 0.383 . Only for $\beta<\beta_{c_{1}}$, it is possible to find a $y_{1}$ (equal to the $t_{1}(\beta)$ of $\S 2$ ) such that $u\left(y_{1}\right)=0$.

For $y>y_{1}, u$ is so small that it is not possible to neglect the left-hand side of (3.8); however, we can neglect $u \ll 1$ in the right-hand sides of (3.8) and (3.3b-d). In order to follow the evolution of $u$ for $y>y_{1}$, we introduce the variable $U=\ln u$, in 
(3.8), that becomes

$$
\alpha^{-1} \frac{d U}{d y}-\left(4 \alpha^{2}\right)^{-1}\left[\frac{d^{2} U}{d y^{2}}+\left(\frac{d U}{d y}\right)^{2}\right]=1-z / \beta,
$$

or defining

$$
\begin{aligned}
& p=\alpha^{-1} \frac{d U}{d y} \\
& p-p^{2} / 4-(4 \alpha)^{-1} \frac{d p}{d y}=1-z / \beta
\end{aligned}
$$

As $\alpha \gg 1$, we can neglect the $O(1 / \alpha)$-term in (3.10b) and thus the solution of the resulting equation, such that $u$ begins to decrease for $y>y_{1}$, is

$$
p=2-2(z / \beta)^{1 / 2}
$$

Coming back to $u$, the solution at this stage is

$$
u(y) / C=\exp \left\{2 \alpha \int_{y_{1}}^{y}(1-\sqrt{z(y) / \beta} d y\},\right.
$$

where the constant $C=O\left(\alpha^{-1 / 2}\right)$ is to be calculated by coupling (3.8)-(3.9) and (3.11) through an intermediate transition stage analogous to the one described in $\S 2$.

Together with (3.11), we must solve the equations

$$
\frac{d v}{d y}=-v, \quad \frac{d w}{d y}=v-w, \quad \frac{d z}{d y}=w-z,
$$

with the initial conditions

$$
v=v_{1}, \quad w=w_{1}, \quad z=\beta, \quad \text { at } y=y_{1},
$$

$v_{1}, w_{1}$ being the values taken by the corresponding solutions of $(3.8 \mathrm{~b}-\mathrm{c})$ at $y=y_{1}$. The equation (3.11) determines $u(y)$ for $y>y_{1}$, while the argument of the exponential is negative. This is the case for $y<y_{2}$, given by

$$
\int_{y_{1}}^{y_{2}}\left\{1-(z(y) / \beta)^{1 / 2}\right\} d y=0
$$

otherwise it is not true that $u \ll 1$. In a third thin transition region, the solution of (3.3) will be described in terms of the variables $\tilde{\eta}=\alpha\left(y-y_{2}\right)$, and it can be approximated by means of the solution of the following equations:

$$
\begin{aligned}
& \frac{d u}{d \tilde{\eta}}-4^{-1} \frac{d^{2} u}{d \tilde{\eta}^{2}}=u(1-u-z / \beta), \\
& v=v_{2}, \quad w=w_{2}, \quad z=z_{2}, \\
& u(-\infty)=0, \quad u(\infty)=1-z_{2} / \beta>0 .
\end{aligned}
$$

In fact, let $t_{2}$ and $x_{2}$ be time and length corresponding to the phase $y_{2}=t_{2}+x_{2} / c$, $c=2 \sqrt{\alpha}, u$ obeys

$$
\frac{\partial u}{\partial \tilde{\tau}}=\alpha \frac{\partial^{2} u}{\partial \tilde{\xi}^{2}}+u(1-z / \beta-u), \quad \tilde{\tau}=\alpha\left(t-t_{2}\right), \quad \tilde{\xi}=\alpha\left(x-x_{2}\right),
$$

while $d v / d \tilde{\tau}=d w / d \tilde{\tau}=d z / d \tilde{\tau}=0$ and therefore $z=z_{2}$ in (3.14g). As $\tilde{\xi} \rightarrow-\infty$, (3.11) 
and (3.13) show that $u(\tilde{\xi}, 0) \sim C \exp \left\{\alpha^{-1 / 2}\left[1-\left(z_{2} / \beta\right)^{1 / 2}\right] \tilde{\xi}\right\}$, so that, according to (3.7), $u(\tilde{\xi}, \tilde{\tau})$ evolves into the wavefront solution of $(3.14 \mathrm{~g})$ with speed $c=2 \sqrt{\alpha}$, as established in $(3.14 \mathrm{a}-\mathrm{f})$. (For the Fisher equation $(3.14 \mathrm{~g})$, the constant $K(c)$ of $(3.7 \mathrm{~b})$ is given by $K(c)=\left\{c / 2-\left(c^{2} / 4-\alpha+\alpha z_{2} / \beta\right)^{1 / 2}\right\} \alpha^{-1}[18]$. Notice that $c=2 \sqrt{\alpha}$ is larger than the minimum allowed velocity $2\left\{\alpha\left(1-z_{2} / \beta\right)\right\}^{1 / 2}$ for $(3.14 \mathrm{~g})$.)

We have thus shown that the speed of the second Fisher wavefront is that of the first one, $2 \sqrt{\alpha}$. After the second wavefront passes, we can again use $(3.3 \mathrm{~b}-\mathrm{d})$ and (3.9) to describe the solution of (3.3) until another value of $y=y_{3}$, such that $u\left(y_{3}\right)=0$, is reached (if $\beta<\beta_{c 2}<\beta_{c 1}$ ). In a similar way to the homogeneous case, if $\beta$ is smaller than a certain $\beta_{c}=0.150$, the solution is a sawtooth type wavetrain moving with the velocity $2 \alpha^{1 / 2}$ of the first Fisher front that becomes periodic for large values of $y$.

For the description of the solution for all values of $\beta$, in the limit $\alpha \rightarrow \infty$, we can proceed in the same form as in the homogeneous case; no detailed description is necessary, in this limit, of the transition regions, that now become discontinuities of $u$ or of $d u / d y$.

For speeds $2 \sqrt{\alpha}$, a wave number $k=\left(3 \alpha^{-1} / 4\right)^{1 / 2}$ corresponds to the frequency $\sqrt{3}$ of the bifurcating time periodic solutions of the diffusive Volterra equation on an unbounded one-dimensional support and, therefore, the bifurcation diagram is similar to the one depicted in Fig. 1 for the homogeneous case (the diffusion term gives vanishingly small contributions of order $\alpha^{-2}$, as shown in Appendix 1). In this case the stability of the wavetrain solution remains an open problem as discussed in $\S 6$.

4. The Volterra equation for continuous delay and small $\beta$. In this section, we shall extend the results of previous sections to the case

$$
\begin{aligned}
& G(\tau)=\sum_{n=p}^{N} g_{n} G^{(n)}(\tau), \quad g_{p}>0, \\
& \int_{0}^{\infty} G^{(n)}(\tau) d \tau=\sum_{n=p}^{N} g_{n}=1, \quad p \geqq 2,
\end{aligned}
$$

and $\beta$ small (in a sense to be made precise later). As we saw at the beginning of $\S 2$, only for $p \geqq 2, u_{e}$ is unstable as $\alpha \rightarrow \infty$ and $\beta \rightarrow 0$.

Defining the variables

$$
v_{n}(x, t)=\int_{0}^{\infty} G^{(n)}(\tau) u(x, t-\tau) d \tau, \quad n=0,1, \cdots, N,
$$

the Volterra equation with kernel (4.1) becomes

$$
\begin{aligned}
& \frac{\partial u}{\partial t}=\alpha u\left(1-u-\beta^{-1} \sum_{n=p}^{N} g_{n} v_{n}\right)+\frac{\partial^{2} u}{\partial x^{2}}, \\
& \frac{\partial v_{0}}{\partial t}=u-v_{0}, \\
& \frac{\partial v_{n}}{\partial t}=v_{n-1}-v_{n}, \quad n=1, \cdots, N .
\end{aligned}
$$

We will solve (4.3) with the initial conditions $u\left(t_{0}\right)=b / \alpha, v_{n}\left(t_{0}\right)=a_{n} / \alpha$ in the homogeneous case and with the boundary conditions (3.4) for the travelling wave problem in the diffusive case.

As in $\S \S 2$ and 3 there is a thin initial region of duration $O\left(\alpha^{-1}\right)$ in $t$ (homogeneous case) or in $y=t+x / c$ (diffusive case) where $u$ rises from 0 to 1 . In this region, (4.3) can be described by means of $(2.8 \mathrm{a})$ or $(3.5 \mathrm{a})$ as in previous sections. 
When $y$ or $t$ are of the order of $1,(4.3)$ becomes

$$
\begin{aligned}
& 1-u-\beta^{-1} \sum_{n=p}^{N} g_{n} v_{n}=0, \\
& \frac{d v_{0}}{d y}=-v_{0}-\beta^{-1} \sum_{n=p}^{N} g_{n} v_{n}+1, \\
& \frac{d v_{n}}{d y}=v_{n-1}-v_{n}, \quad n=1,2, \cdots, N,
\end{aligned}
$$

with the initial conditions

$$
1-u=v_{n}=0, \quad n=0,1, \cdots, N \quad \text { at } y=0 .
$$

In these equations, $y=t$ in the homogeneous case and $y=t+x / c$ when diffusion is present.

Let us suppose now that

$$
\beta^{1 /(p+1)} \ll 1
$$

so that, with the scaling

$$
\begin{aligned}
& \eta=\beta^{-1 /(p+1)} y, \\
& v_{n}=\beta^{(n+1) /(p+1)} W_{n},
\end{aligned}
$$

equations (4.4)-(4.5) become

$$
\begin{aligned}
& u=1-g_{p} W_{p}, \\
& \frac{d W_{0}}{d \eta}=1-g_{p} W_{p}, \\
& \frac{d W_{n}}{d \eta}=W_{n-1}, \quad n=1, \cdots, N, \\
& W_{n}(0)=0, \quad n=0,1, \cdots, N .
\end{aligned}
$$

The solution of the linear system (4.7) shows that $u=0$ for a certain value $\eta_{1}$ such that

$$
\sum_{j=0}^{p}\left(\zeta^{-1}\right)_{j 0} e^{\zeta \eta_{1}}=0, \quad \zeta_{j}=g_{p}^{1 /(p+1)} \exp \left\{\frac{\pi(2 j+1) i}{p+1}\right\},
$$

where $\left(\zeta^{-1}\right) i j$ is the inverse matrix of $\zeta_{i j}=\left(\zeta_{j}\right)^{i}, i, j=0,1, \cdots, p$. If $g_{n}=\delta_{n 2}$, (4.8) gives

$$
W_{n}\left(\eta_{1}\right) \equiv W_{n}^{1}, \quad \eta_{1}=1.851, \quad W_{0}^{1}=1.377, \quad W_{1}^{1}=1.536
$$

Clearly, as $y \rightarrow y_{1}=\eta_{1} \beta^{1 /(p+1)}, u$ becomes small so that $\alpha u \gg 1$ is no longer true. After $y_{1}, u \ll 1$ and therefore we can approximate the Volterra equation by

$$
\frac{d u}{d y}=\alpha u\left(1-\beta^{-1} \sum_{n=p}^{N} g_{n} v_{n}\right)
$$

in the homogeneous case, or by

$$
\frac{d u}{d y}=2 \alpha u\left[1-\left(\beta^{-1} \sum_{n=p}^{N} g_{n} v_{n}\right)^{1 / 2}\right]
$$


in the diffusive case (cf. $\S 3$ ), while the $v_{n}$ 's evolve in both cases according to the equations

$$
\frac{d v_{0}}{d y}=-v_{0}, \quad \frac{d v_{n}}{d y}=v_{n-1}-v_{n}, \quad n=1, \cdots, N .
$$

The initial conditions are

$$
v_{n}\left(y_{1}\right)=W_{n}^{1} \beta^{(n+1) /(p+1)}, \quad n=0, \cdots, N .
$$

As $\beta^{1 /(p+1)} \rightarrow 0$, the solution of the linear ODE system (4.11)-(4.12) approaches

$$
v_{n}(y)=W_{0}^{1} \beta^{1 /(p+1)}\left(y-y_{1}\right)^{n} e^{-\left(y-y_{1}\right)}(n !)^{-1},
$$

and therefore $u(y)$ will be

$$
u(y)=C e^{-\alpha I\left(y-y_{1}\right)}
$$

where

$$
I(s)=\int_{0}^{s}\left\{\beta^{-p /(p+1)} W_{0}^{1} \sum_{n=p}^{N} g_{n} \frac{s^{n}}{n !} e^{-s}\right\} d s-s,
$$

in the homogeneous case, or

$$
\frac{I(s)}{2}=\beta^{-(p /(p+1)) / 2} \sqrt{W_{0}^{1}} \int_{0}^{s}\left\{\sum_{n=p}^{N} g_{n} \frac{s^{n}}{n !} e^{-s}\right\}^{1 / 2} d s-s,
$$

in the diffusive case. The constant $C$ must be calculated through an intermediate stage analogous to the one described in $\S 2$ and the result is

$$
C=\left(\frac{W_{p-1}^{1}}{2}\right)^{1 / 2} r\left(\alpha \beta^{1 /(p+1)}\right)^{-1 / 2},
$$

where

(4.15b-c) $\quad r=g_{p}^{1 / 2}$ (homogeneous), $\quad r=\left(g_{p} / W_{p}^{1}\right)^{1 / 4}$ (diffusive).

Consistency requires $C \rightarrow 0$ as $\alpha \rightarrow \infty$, and therefore it must be

$$
\alpha^{-1} \ll \beta^{1 /(p+1)} \ll 1 .
$$

Equation (4.14) is a good approximation to $u$ while $I>0$, and therefore after a certain $y_{2}$ such that $I\left(y_{2}-y_{1}\right)=0, u \ll 1$ is no longer true and we should describe $u$ by $(4.4 \mathrm{a}-\mathrm{c})$ with initial conditions given by $v_{n}\left(y_{2}\right)$ of $(4.13)$. As $\beta \rightarrow 0$, it may be expected that $y_{2}$ corresponds to a large $s$ in (4.14), and therefore we can approximate $(4.14 \mathrm{~b}-\mathrm{c})$ by taking the upper limit of the integrals equal to $\infty$ :

$$
I(s)=\beta^{-p /(p+1)} W_{0}^{1}-s \quad \text { (homogeneous), }
$$

After $y_{2}=O\left(\beta^{-p(p+1)^{-1 /(2)}}\right)$, (where (2) is 1 in the homogeneous case and 2 in the diffusive case), a stage described by $(4.4 \mathrm{a}-\mathrm{c})$ begins. If the $v_{n}\left(y_{2}\right)$ 's in (4.13) are $O\left(\beta^{(n+1) /(p+1)}\right)$, this new stage coincides with the one described by $(4.7 \mathrm{a}-\mathrm{d})$ and $u$ is periodic with period $O\left(\beta^{-p(p+1)^{-1 /(2)}}\right)$. After some algebra, it is possible to see that this consistency requirement is not true for integers in a neighborhood of

$$
n_{*}=p(p+1)^{-1} \beta^{-(p /(p+1)) / 2},
$$


and therefore our kernel $G(\tau)$ should fulfill

$$
g_{n} \neq 0 \text { only if }\left|n-n_{*}\right| \gg 1 .
$$

In particular, condition (4.18b) is satisfied if $N$ is finite and

$$
\beta \ll 1 / N .
$$

\section{Volterra equation with discrete delay.}

5.1. Homogeneous oscillations. As we shall show in this section, the homogeneous limit cycle of the Volterra equation with discrete delay has a square form and is reached after a short transient stage, in contradistinction with the sawtoothlike limit cycle in the case of continuous delay. The same difference exists between the wavetrains when diffusion is included.

Since the method of solution is the same as in previous sections, we shall only outline the results.

The steady solution $u_{e}$ of the homogeneous equation

$$
\frac{d u}{d t}=\alpha u\left\{1-u-\beta^{-1} u(t-1)\right\}
$$

is A.S. if $\alpha<\alpha_{0}, \alpha_{0}$ running from $\pi / 2$ when $\beta=0$ to infinity when $\beta=1$. For $\beta>1$, $u_{e}$ is always an A.S. solution of (5.1), whereas for $\beta<1$ a phase A.S. limit cycle bifurcates from $\alpha=\alpha_{0}$. See reference [2]. The analysis given here corresponds to $\alpha \gg 1$ and $\beta>0$.

Let us assume that $u \ll 1$ if $-1 \leqq t \leqq 0$. In this case, we can neglect the delayed term in (5.1) when $0<t<1$ and, if $0<t \ll 1$, we can also neglect the instantaneous regulatory term, $-\alpha u^{2}$. As a result, $u$ will grow exponentially and will reach the value $\frac{1}{2}$ at a time $t_{0}$ that depends on $u(0)$. To fix ideas, if $u(t)$ is an increasing function of $t$ in the interval $[-1,0]$ and $u(0)=O\left(e^{-\alpha}\right), t_{0}=O(1)$; if $u(0)=O\left(\alpha^{-p}\right) p>0, t_{0}=$ $O\left(\alpha^{-1} \ln \alpha\right)$; and if $u(0)=O(1), t_{0}=O(1 / \alpha)$. In any case, we can translate the origin of time in such a way that $u(0)=\frac{1}{2}$, and $u \sim e^{\alpha t}$ as $\alpha t \rightarrow-\infty$; then the reduced equation

$$
\frac{d u}{d t}=\alpha u(1-u), \quad u(0)=\frac{1}{2},
$$

holds valid in a certain interval around $t=0$ whose lower extreme is $-t_{0}$ and whose higher extreme is to be determined. The solution of $(5.2 \mathrm{a})$ is

$$
u(t)=e^{\alpha t} /\left(1+e^{\alpha t}\right) .
$$

After a certain instant $t_{1} \in(0,1), u(t-1)$ given by $(5.2 \mathrm{~b})$ is no longer small and we have to add the term $-u(t-1) / \beta$ given also by $(5.2 \mathrm{~b})$ to $(5.2 \mathrm{a})$. The result is that $u(t)=\tilde{u}(\tau)$ satisfies

$$
\begin{aligned}
& \frac{d \tilde{u}}{d \tau}=\tilde{u}\left\{1-\tilde{u}-\beta^{-1} e^{\tau} /\left(1+e^{\tau}\right)\right\}, \\
& \tau=\alpha(t-1) .
\end{aligned}
$$

Equation (5.3a) must be solved with the condition

$$
\lim _{\tau \rightarrow-\infty} \tilde{u}(\tau)=1,
$$

so that the solution matches with the one given by $(5.2 \mathrm{~b})$.

In the overlap domain between the approximations given by (5.2) and (5.3), the maximum of the oscillation is reached. A simple linearization of (5.3a) around $u=1$ 
gives us the approximated location of the maximum $t=t_{\max }$,

$$
t_{\max }=\frac{1}{2}+(2 \alpha)^{-1} \ln (2 \beta) \text {, }
$$

an also an approximation to the solution of (5.3a) around it

$$
u(t)=1-e^{-\alpha t}-e^{\alpha t-\alpha} /(2 \beta) .
$$

According to (5.3a), the asymptotic behavior of $\tilde{u}(\tau)$ is given by

$$
\tilde{u}(\tau) \sim \tilde{c}_{1}(\beta) \exp \left\{\left(1-\beta^{-1}\right) \tau\right\} \quad \text { for } \tau \rightarrow \infty .
$$

If $\beta<1, u$ will tend to zero as $\tau \rightarrow+\infty$, but, near $t=t_{1}+1,(5.2 \mathrm{~b})$ ceases to be a good approximation to $u(t-1) / \beta$, that must be substituted by $\tilde{u}(\tau-\alpha)$; instead we have another stage at which the solution of (5.1) can be described by $u(t)=u^{*}(\sigma)$, with

$$
\begin{aligned}
& \frac{d u^{*}}{d \sigma}=u^{*}(1-\tilde{u}(\sigma) / \beta), \\
& \sigma=\alpha(t-2) .
\end{aligned}
$$

The matching condition at this stage is

$$
\lim _{\sigma \rightarrow-\infty} u^{*}(\sigma)=\tilde{c}_{1}(\beta) \exp \left\{\left(1-\beta^{-1}\right)(\sigma+\alpha)\right\} .
$$

If we define a new variable $v(\sigma)$ as

$$
v(\sigma)=\ln \left\{u^{*}(\sigma) /\left[\tilde{c}_{1}(\beta) e^{\left(1-\beta^{-1}\right) \alpha}\right]\right\},
$$

(5.6) can be written as

$$
\begin{aligned}
& \frac{d v}{d \sigma}=1-\tilde{u}(\sigma) / \beta \\
& \lim _{\sigma \rightarrow-\infty} v(\sigma)=\left(1-\beta^{-1}\right) \sigma .
\end{aligned}
$$

In this stage, the minimum of the oscillation, $v_{\min }(\beta)$, is attained and $u$ increases afterwards so that

$$
v(\sigma)=\sigma+\ln \tilde{c}_{2}(\beta) \quad \text { as } \sigma \rightarrow \infty .
$$

Equation (5.9) corresponds to

$$
u^{*} \sim e^{\alpha[t-T(\beta)]}
$$

with

$$
T(\beta)=1+1 / \beta-\alpha^{-1} \ln \left[\tilde{c}_{1}(\beta) \tilde{c}_{2}(\beta)\right]
$$

$u^{*}$ in (5.10) has the same asymptotic form as $u \sim e^{\alpha t}$ in $(5.2 \mathrm{~b})$ when $t \ll 1$. Therefore, we can consider that, after this first relaxation oscillation, a periodic solution of (5.1) with period $T(\beta)$ has been reached.

Inserting (5.8a) into (5.3a) and performing the integration we obtain a relation between $u$ and $v$,

$$
\ln \tilde{u}=\sigma+\beta(v-\sigma)-\beta^{-1} \ln \left(1+e^{\sigma}\right)+\text { const. }
$$

The constant of integration can be evaluated using (5.3c) and (5.8b) and is equal to zero. If we now take the limit $\alpha \rightarrow \infty$ in that expression and use (5.5) and (5.9), we 
arrive at

$$
\tilde{c}_{1}=\tilde{c}_{2}^{\beta}
$$

with

$$
\tilde{c}_{2}(\beta)=\lim _{\sigma \rightarrow \infty}\left\{\int_{-\infty}^{\sigma}[1-\tilde{u}(\sigma) / \beta] d \sigma-\sigma\right\},
$$

being the constant in (5.9).

In the limit $\alpha \rightarrow \infty$, the periodic solution of (5.1) thus obtained has an asymptotic square form and each period is composed of two very different parts joined by abrupt transitions. During a unit of time, $u$ is very close to 1 , and after this stage, it suddenly falls to zero and remains so during a time $1 / \beta$. From our construction it is clear that the limit cycle is phase A.S. The numerical values of $\tau_{1}(\beta), v_{\min }(\beta), c_{1}(\beta)$, and $c_{2}(\beta)$ are gathered in Table 1 calculated by direct numerical integration of (5.3) and (5.8),

TABLE 1

\begin{tabular}{ccccc}
\hline$\beta$ & \multicolumn{1}{c}{$\tau_{1}(\beta)$} & $v_{\min }(\beta)$ & $c_{1}(\beta)$ & $c_{2}(\beta)$ \\
\hline 0.1 & 1.40847 & 1.75741 & $3.489 \times 10^{10}$ & 0.999491 \\
0.2 & 1.11537 & 1.66206 & 5120 & 0.999916 \\
0.3 & 0.807008 & 1.58380 & 56.17 & 0.999992 \\
0.4 & 0.443183 & 1.49564 & 6.889 & 1.000000 \\
0.5 & $1.907 \times 10^{-6}$ & 1.38628 & 2 & 0.999992 \\
0.6 & -0.54944 & 1.24754 & 0.8479 & 1.000002 \\
0.7 & -1.25036 & 1.0708 & 0.4258 & 1.0001 \\
0.8 & -2.20492 & 0.843181 & 0.2216 & 1.00378 \\
0.9 & -3.74772 & 0.536887 & 0.9681 & 1.00112 \\
\hline
\end{tabular}

so that an excellent correlation of the results can be obtained by writing $c_{2}=1$. The constants $c_{1}(\beta)$ and $c_{2}(\beta)$ in Table 1 are related to $\tilde{c}_{1}(\beta), \tilde{c}_{2}(\beta)$ by the formulas

$$
\begin{aligned}
& c_{1}(\beta)=\tilde{c}_{1}(\beta)[\beta /(1-\beta)]^{1-\beta^{-1}}, \\
& c_{2}(\beta)=\tilde{c}_{2}(\beta)[\beta /(1-\beta)]^{\beta^{-1}} .
\end{aligned}
$$

Inserting (5.12) with $c_{2}=1$ into (5.11), we arrive at the following result:

$$
T(\beta)=1+1 / \beta-\alpha^{-1}(1+1 / \beta) \ln \{(1-\beta) / \beta\} .
$$

In the case $\beta>1$, the solution of (4.3a) gives $\tilde{u} \rightarrow 1-1 / \beta$ for large $\tau$; so that for large $\alpha, \tilde{u}$ jumps from 0 to 1 at $t=0$ and then it retains the value 1 in the interval $(0,1)$, and jumps to $1-1 / \beta$ at $t=1$. A similar analysis to the one given above shows that $u$ retains the value $1-1 / \beta$ in the interval $(1,2)$, jumping at $t=2$ to the value $1-1 / \beta+1 / \beta^{2}$, that is kept in the interval $(2,3)$, and so on, until, after an infinite number of jumps, the steady state value $1-1 / \beta+1 / \beta^{2}-\cdots=u_{e}=\beta /(1+\beta)$, is reached (Fig. 3).

5.2. Nonhomogeneous oscillations. Let us now find the travelling wave solutions of the diffusive Volterra equation. As in (3.7a) the first Fisher wavefront satisfies

$$
\begin{aligned}
& u(\eta)=-a_{0} \eta c^{2 \eta} \quad \text { for } \eta \rightarrow-\infty, \\
& u(\eta)=1-a_{1}(\beta) e^{-2\left(2^{1 / 2}-1\right) \eta} \text { for } \eta \rightarrow \infty,
\end{aligned}
$$




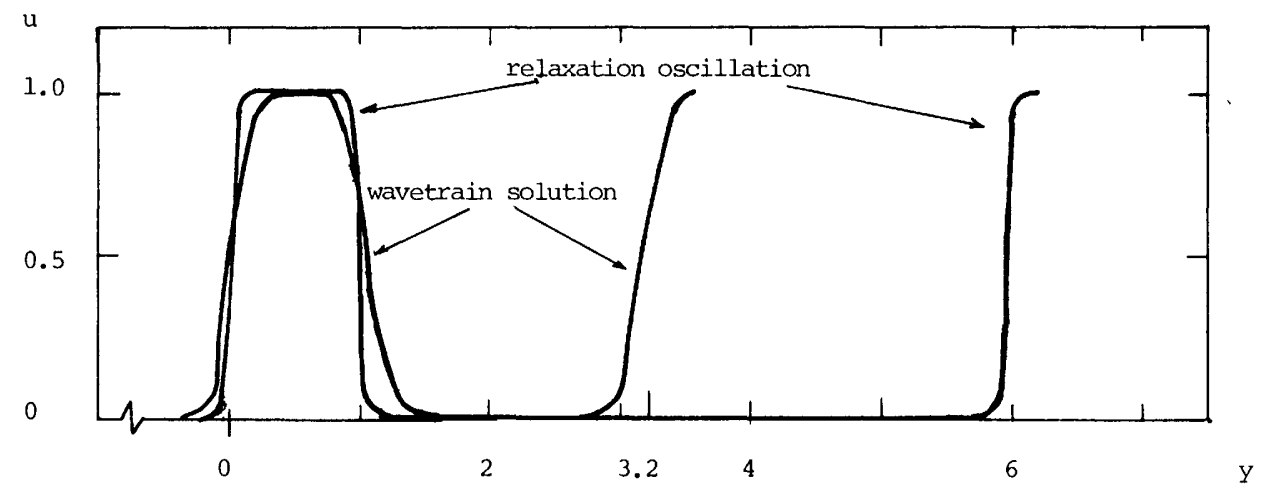

FIG. 3a. Periodic solution for $\beta=0.2$ and discrete delay.

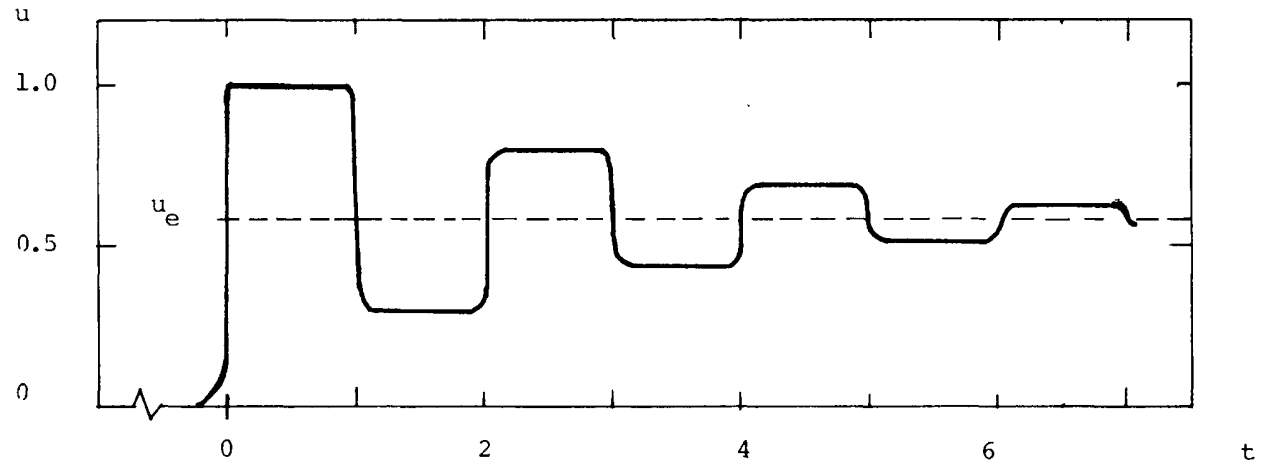

FIG. 3b. Solution of the nondiffusive equation for $\beta=1.4$, and discrete delay. The steady state $u_{e}=0.59$ is A.S.

$a_{0}>0$ being a constant characteristic of the Fisher wavefront (see [25, Thm. 2.2]) and $a_{1}(\beta)$ being an integration constant such that (5.14a) holds true. For $y=t+x / c \sim 1$, we have to add the delay term as we did in the homogeneous case. Since the process is the same as used in the case without diffusion we shall only provide the results. It is necessary to simultaneously solve the following system of equations:

$$
\begin{aligned}
& \frac{d u}{d \eta}-\lambda \frac{d^{2} u}{d \eta^{2}}=u(1-u), \quad \lambda=\frac{1}{4}, \\
& \frac{d v}{d \eta}-\lambda \frac{d^{2} v}{d \eta^{2}}=v(1-v-u / \beta), \\
& \frac{d w}{d \eta}-\lambda \frac{d^{2} w}{d \eta^{2}}=w(1-v / \beta),
\end{aligned}
$$

with the initial conditions

$(5.16 \mathrm{a}-\mathrm{c}) \quad u(\eta)=-a_{0} \eta e^{2 \eta}, \quad v=1, \quad w=\exp \left\{-2\left(\beta^{-1 / 2}-1\right) \eta\right\} \quad$ for $\eta \rightarrow-\infty$.

For $\eta \rightarrow \infty$, the asymptotic form of the solutions of (5.15)-(5.16) is

$(5.17 \mathrm{a}-\mathrm{c}) \quad u=1-a_{1}(\beta) e^{-2\left(2^{1 / 2}-1\right) \eta}, \quad v=a_{1}(\beta) a_{2}(\beta) e^{-2\left(\beta^{-1 / 2}-1\right) \eta}, \quad w=a_{3}(\beta) \eta e^{2 \eta}$. 
The solution of the diffusive Volterra equation with discrete delay is $u=u(\alpha y)$ if $y \in(0,1), u=v(\alpha y-\alpha)$ in the second stage around $y=1$, and $u=w(\alpha y-2 \alpha)$ around $y=2$ in the last stage of a period. The period of the wavetrain is then

$$
T(\beta)=1+\beta^{-1 / 2}-(2 \alpha)^{-1} \ln \left\{a_{1}(\beta) a_{2}(\beta) a_{3}(\beta)\right\} .
$$

As a first approximation, the wavetrain has a square form. Each period has a part in which $u=1$ of duration $t=1$, followed by another part in which $u=0$ during a longer time $\beta^{-1 / 2}$.

6. Discussion. Hitherto, most of the results concerning delay-diffusional equations were local (i.e., they issued from studies of the influence of diffusion on steady states or limit cycles that are stable solutions of the corresponding nondiffusional equations [1], [2], [3], [12], [21], [22]), and extended well-known results for reaction-diffusion equations [4]. In this paper, we have taken advantage of the large magnitude of the dimensionless delay $\alpha$ to construct the homogeneous limit cycle and a variety of wave-like solutions using the method of matched asymptotic expansions. We have checked the method for the nondiffusive Volterra equation without predators (Hutchinson delayed logistic equation, $K=\infty$, or more precisely, $\beta e^{\alpha-1} \ll 1$, in (1.2), [15], [16]) and comparison with Jones' paper [8] proves that the solution obtained by matched asymptotic expansions is a higher bound to the true solution and an increasingly precise approximation as $\alpha$ increases. In this fashion, more accurate approximations could be constructed following Fowler's method [5] (i.e., using $e^{\alpha t}$ as a fast-varying time variable), but the results would have remained basically unchanged.

Concerning the stability of the homogeneous limit cycle as a solution of the diffusive Volterra equation, a theorem by Maginu can be of some help [14]. In fact, the limit cycle solution of $\S \S 2,4$ and 5 is a phase A.S. solution of the homogeneous equation as can be seen from our construction. All the corresponding Floquet exponents of the homogeneous limit cycle therefore have negative real parts except a single one that is zero (there are four of such exponents if the delay is continuous with $g_{n}=\delta_{n 2}$ and an infinite number if the delay is discrete). Using the Floquet theory for equations with delay [6] and following step by step Maginu's proof, it is possible to demonstrate a similar statement, namely:

"If $u(t)$ is a phase A.S. time periodic solution (in the absence of diffusion), of period $T$, of the following equation,

$$
\frac{\partial u}{\partial t}=\frac{\partial^{2} u}{\partial x^{2}}+f\left(u_{t}\right), \quad u_{t}(\theta)=u(t+\theta), \quad-r \leqq \theta \leqq 0, \quad-\infty<x<\infty,
$$

where $r=1$ for discrete delay and $r=\infty$ for continuous delay, $u(t)$ will be an unstable solution of $(6.1)$ if $T^{\prime}(0)<0$, and will be lineally stable to large wavelength disturbances if $T^{\prime}(0)>0 ; T(\nu)$ being the period of the time periodic solution of the following equation

$$
(1+\nu) \frac{\partial \psi}{\partial t}=f\left(\psi_{t}(\cdot, \nu)\right), \quad \nu>0
$$

that satisfies $\psi(t, 0)=u(t), T(0)=T$; and $T^{\prime}(\nu)=d T / d \nu^{\prime \prime}$. Applying this result to the homogeneous oscillation of $\S 5$, we obtain

$$
T(\nu)=1+1 / \beta-(1+\nu)(1+1 / \beta) \alpha^{-1} \ln \{(1-\beta) / \beta\},
$$

and therefore have

$$
T^{\prime}(0)=-\alpha^{-1}(1+1 / \beta) \ln \{(1-\beta) / \beta\} .
$$


Hence, the homogeneous limit cycle is unstable to inhomogeneous disturbances if $0<\beta<\frac{1}{2}$, and lineally stable to large wavelength disturbances if $\frac{1}{2}<\beta<1$. The same result was obtained near the bifurcation point using multiscale techniques [2]. Maginu's theorem is also applicable to the homogeneous limit cycle of $\S 2$ (continuous delay) but it would require determination of the following order of approximation in the expansions to calculate $o(1)$ terms in the period.

In relation to the stability of the periodic wavetrain solutions of $\S \S 3$ to 5 , we have not been able to find out a similar criterion; even though, our asymptotic analysis, together with the asymptotic stability properties of Fisher wavefronts, suggests that these wavetrains should be stable.

For the Volterra equation, with a sufficient initial stimulus, one (or many) pulse can be initiated which propagates throughout the medium, so that the medium on which the Volterra equation holds valid is excitable (for sufficiently small $\beta$ ) [9].

We shall see now that the Volterra equation may give rise to pacemaker and leading center appearance, thus presenting the main features of excitable media.

Pacemakers and leading centers are spatial regions in which travelling wavetrains are originated and from which the wavetrains spread out through the medium. The difference is that a pacemaker is a spatial inhomogeneity in which the governing equations are different from the equations in the rest of the space, while in a leading center the governing equations are the same as in the rest and the waves are created by a special initial condition [7], [20], [24].

Let us consider the simple situation in which a virgin space (where the Volterra equation holds true) is encroached from a reservoir where food is superabundant (so that there is no delay term in the Volterra equation: $\beta^{-1}=0, u=1$ for all $t$ on $x>0$ ):

$$
\begin{array}{lll}
u(x, t)=0 & \text { for } t \leqq 0 & \text { if } x<0 \\
u(x, t)=1 & \text { for all } t & \text { if } x>0 .
\end{array}
$$

The first wavefront is initiated in a region of width $O\left(\alpha^{-1 / 2}\right)$ around $x=0$; there $u$ abruptly grows from 0 to 1 in a time $O\left(\alpha^{-1}\right)$. As a first approximation the solution of (1.11) and (6.5) satisfies (3.6) (no matter the kind of delay we consider) and a Fisher wavefront advances toward the left with velocity $2 \alpha^{1 / 2}$. The region $x>0$ therefore behaves like a pacemaker emitting a finite number of wavefronts if $\beta>\beta_{c}$ or a periodic wavetrain if $\beta<\beta_{c}\left(\beta_{c}=1\right.$ for discrete delay and $\beta_{c}=0.150$ for continuous delay with $g_{n}=\delta_{n 2}$ ).

With respect to the leading center phenomenon, let us assume the following initial condition:

$$
\begin{aligned}
& u(x, t)=0 \quad \text { for all } x \text { for all } t<0, \\
& u(x, t)= \begin{cases}0 & \text { for }|x| \geqq x_{0} \alpha^{-1 / 2} \text { at } t=0, \\
u_{0}(x) \in C^{1}[0,1] & \text { for }|x|<x_{0} \alpha^{-1 / 2} \text { at } t=0,\end{cases}
\end{aligned}
$$

with $0<x_{0}=O(1)$.

Irrespective of the kind of delay considered, $u$ obeys Fisher's equation

$$
\begin{aligned}
& \frac{\partial u}{\partial \tau}=\frac{\partial^{2} u}{\partial \tilde{x}^{2}}+u(1-u), \\
& u(\tilde{x}, 0)= \begin{cases}0 & \text { for }|\tilde{x}| \geqq x_{0}, \\
u_{0}(x) & \text { for }|\tilde{x}|<x_{0},\end{cases} \\
& \tau=\alpha t, \quad \tilde{x}=\alpha^{1 / 2} x,
\end{aligned}
$$


and as $\tau \rightarrow \infty, u$ evolves into a pair of diverging wavefronts (moving at the Fisher wavespeed $\tilde{c}=2$ ): for $\tilde{x}>0 u$ tends (uniformly in $\tilde{x}$ ) to a Fisher wavefront moving toward the right and for $\tilde{x}<0 u$ uniformly approaches a Fisher wavefront moving toward the left ([25], Theorem 8.1). Therefore, for $\tilde{x}>0$, the analysis given in $\S \S 3$, 4 and 5 shows that there exists a wavetrain (with wavespeed $2 \sqrt{\alpha}$ in the original variables $x, t)$ moving toward the right and, for $\tilde{x}<0$, another Fisher wavetrain advances toward the left. The point $x=0$ is therefore a leading center if $\beta<\beta_{c}$, and only emits a finite number of pulses if $\beta>\beta_{c}{ }^{1}$

It is interesting to compare the wave-like solutions of the Volterra equation (wavetrains, pacemakers and leading center) with the corresponding solutions of reaction-diffusion models of excitable media [9], [7], [20], [24]. As in reaction-diffusion equations, the velocity of the successive wavefronts depends only on reaction and diffusion (the velocity is proportional to the square root of the birth rate, $r$, multiplied by the diffusivity, $D$, in dimensional units), and it does not depend on the delay, although the delay is responsible for the creation of wavefronts that follow the first one, solution of Fisher's equation. On the other hand, if we have, for example, the pacemaker initial condition (6.5), the first wavefront keeps the velocity of the following ones equal to its own velocity, $2 \alpha^{1 / 2}$, that is larger than the minimum allowed velocity for the successive fronts, as was pointed out in $\S \S 3,4$ and 5. Using the terminology of Tyson and Fife's paper [24], the first wavefront emitted by a pacemaker is a "trigger" one whereas the successive fronts are "phase" waves. For reaction-diffusion two-components models, [9], [24], the velocity of the resulting wavetrain emitted by a pacemaker is different (usually smaller) from the velocity of the first front, and at least the first, third, etc. wavefronts are "trigger" waves, whereas the second, fourth, etc. fronts may [24] or may not [9] be "phase" waves.

Finally, it should be noted that the spruce budworm equation

$$
\frac{\partial u}{\partial t}=\frac{\partial^{2} u}{\partial x^{2}}+\alpha u\left\{1-Q^{-1} \int_{0}^{\infty} G(\tau) u(x, t-\tau) d \tau-R^{-1} u /\left(1+u^{2}\right)\right\}
$$

with realistic values of the dimensionless parameters, i.e., $R=0.994, Q=302, \alpha \sim 10$ (see [22] and references quoted therein), is reduced to the Volterra equation with $\beta \rightarrow 0$, because the saturation term $u^{2} /\left(1+u^{2}\right)$ is uniformly small, of the order $O\left(Q^{-1}\right)$, when the change of variable $u / Q=v$ is carried out in (6.8). In this fashion, wave-like phenomena like the ones described in this paper should be expected, with relatively short outbreak periods (in which $u / Q \sim 0$ ), followed by long endemic periods (in which $u / Q \sim 0$ ). This situation resembles the one reported in the literature before the extensive insecticide management policy, even though quantitative agreement is far from being satisfactory [23].

Appendix 1. Hopf bifurcation in the limit $\alpha \rightarrow \infty$. As $\alpha \rightarrow \infty$, the homogeneous Volterra equation (for $g_{n}=\delta_{n 2}$ ) has a bifurcating branch of time-periodic solutions at $\beta_{0}=0.125\left(1+27 \alpha^{-1}\right)+O\left(\alpha^{-2}\right)$ as can be seen from the linear stability analysis around $u_{e}=\beta /(1+\beta)$. The pair of simple eigenvalues, $\pm i \nu_{0}=\lambda\left(\beta_{0}^{-1}\right), \nu_{0}=$ $\sqrt{3}+O\left(\alpha^{-1}\right), \quad$ crosses the imaginary axis with speed $d \lambda(8) / d \beta^{-1}=$ $-8(1+i \sqrt{3}) / 3+O\left(\alpha^{-1}\right)$ (we will use $\beta^{-1}$ as our bifurcation parameter). The inclusion of the diffusive term does not change this picture because it only adds terms proportional to $k^{2} \alpha^{-1}=\frac{3}{4} \alpha^{-2}$ ( $\lambda$ in the bifurcation problem of periodic wavetrains travelling with the Fisher wavespeed $2 \sqrt{\alpha})$ in the formulas for $\beta_{0}$ and $\nu_{0}$.

If we call $\lambda=\beta^{-1}-\beta_{0}^{-1}, U=\left(u-u_{e}, v_{0}-u_{e}, v_{1}-u_{e}, v_{2}-u_{e}\right)$, a standard LyapunovSchmidt procedure [28] tells us that there is a unique branch of periodic solutions 
bifurcating from $u_{e}$ of the form

$$
(\lambda, U)=\left(\lambda, x_{1} \phi_{0}+W\left(\lambda, x_{1} \phi_{0}\right)\right),
$$

where $\lambda$ is a locally convergent series parametrized by $x_{1} \in \mathbb{R}, \phi_{0}=\operatorname{Re}\left[\psi e^{-i \nu_{0} t}\right], \psi$ is the eigenvector associated to $i \nu_{0}$, and $W$ is the solution of the original equation projected onto the complementary subspace of $\operatorname{span}\left[\phi_{0}\right] . x_{1}$ is a solution of the following reduced bifurcation equation,

$$
\sum_{j, 1=0}^{\infty} C_{i 1} \lambda^{j} x_{1}^{l}=0, \quad C_{00}=0 .
$$

In (A.2), $C_{j 0}=\operatorname{Re}\left(d \lambda(8) / d \beta^{-1}\right)=-8 / 3$ if $C_{10}=\cdots=C_{j-1,0}=0$ and $C_{j 0} \neq 0$. It is possible to calculate the coefficients $C_{j 1}$ using the recursion formulas given by Kielhöfer [28] with the result that $C_{j, 2 l+1}=0, C_{j, 21}=O\left(\alpha^{-1}\right)$ and therefore, as $\alpha \rightarrow \infty$, the branch of periodic solutions bifurcates vertically and a local calculation does not make sense.

As $\beta$ crosses the bifurcation point, only a single pair of simple complex conjugate eigenvalues crosses to the complex right half plane. Hence, the global Hopf bifurcation theorem [26] tells us that the branch of periodic solutions $k=\{(T, a, \beta)\}$ ( $T$ being period and $a$ an initial condition such that $U(T, a, \beta)=a)$ that bifurcates from $u_{e}$ at $\beta_{0}$ is connected and that either:

(1) $k$ contains a point $(T, a, \beta) \neq\left(2 \pi / \nu_{0}, 0, \beta_{0}\right)$, where $T>0$ and $(a, \beta)$ is a steady state of the ODE system; or

(2) $k$ contains points $(T, a, \beta)$ with $T+|a|+|\beta|$ arbitrarily large.

The Volterra equation has only two steady states: $u=v_{n}=0$ (which has eigenvalues -1 (of multiplicity 3 ) and $\alpha$ ) and $u=v_{n}=u_{e}$ (which only has a Hopf bifurcation point at $\beta_{0}=\frac{1}{8}$ as $\alpha \rightarrow \infty$ ). For these steady states, we can disregard the possibility (1) as no Hopf point different from $\left(2 \pi / \nu_{0}, 0, \beta_{0}\right)$ exists.

The branch $k$ cannot obviously exist for $\beta \rightarrow \infty$ where the delay term disappears. On the other hand, our construction in $\S 2$ shows that $0 \leqq u \leqq 1, v_{n}$ bounded, after a time $O\left(\alpha^{-1}\right)$ for any initial condition $u>0, v_{n}>0$ as $\alpha \rightarrow \infty$. For finite $\alpha\left\{u>0, v_{n}>0\right\}$ is an invariant region and no solution can become unbounded in finite time ([27, Lemma 5]). As a consequence, the branch $k$ must contain points with $|a|$ and $\beta$ bounded and $T$ arbitrarily large. Let us prove now that only the periodic solution described in $\S 2$ has these properties and therefore the relaxation oscillation is part of $k$ as shown in Fig. 1.

With $|a|$ and $\beta$ bounded, the only possibility for branch $k$ to have $T \rightarrow \infty$ is forming a saddle loop. The steady state $u=v_{n}=u_{e}$ cannot have a saddle loop (for $\beta>\beta_{0}$ it is stable and for $\beta<\beta_{0}$ no nonconstant solution of the Volterra equation tends to $u_{e}$ in the invariant region according to [27, Lemma 12]). If we assume that $u=v_{n}=0$ has a saddle loop and take initial conditions on the outgoing part of the loop very close to $u=v_{n}=0$, the construction of $\S 2$ shows us that, for $\beta<\beta_{c}$, we reach the relaxation oscillation with period $T$ and that $T \rightarrow \infty$ as $\beta \rightarrow 0$. QED

Notice that the existence of a periodic solution for, at least, $0<\beta<\beta_{0}$ was proven in [27] for any $\alpha>0$.

${ }^{1}$ More precisely, the phase shift $x^{*}$ in the Fisher wavefronts depends on the initial condition $u_{0}(x)$ so that $x^{*}=O(1 / c)=O\left(\alpha^{-1 / 2}\right)\left(\left[25\right.\right.$, Thm. 9.4]) and therefore for each $u_{0}(x)$ the center of the pattern, i.e., the point equidistant from both Fisher wavefronts will be a certain $x_{1}=\frac{1}{2}\left(x_{\text {right }}^{*}+x_{\text {left }}^{*}\right),\left|x_{1}\right|<x_{0} \alpha^{-1 / 2}$ for all $t$. The same should be true for a more general initial condition (6.6) with $x_{0}=O(1)$ instead of $x_{0} \alpha^{-1 / 2}$. As $\alpha \rightarrow \infty$ the point $x=0$ would be the leading center. 
Acknowledgments. We are indebted to Mr. Francisco J. Higuera for his valuable discussions and numerical computations, and to the anonymous referee for useful comments and suggestions.

\section{REFERENCES}

[1] L. L. Bonilla AND M. G. Velarde, Time delay, diffusion and advection in a model for the time evolution of a spruce budworm population, J. Interdiscipl. Cycle Res., 12 (1981), pp. 267-272.

[2] - Systems with time delay: A model of relevance in ecology and related sciences, presented at the 7th International Conference on System Dynamics, Brussels, Belgium, June, 1982.

[3] D. S. COHEN AND S. ROSENBLAT, Multispecies interactions with hereditary effects and spatial diffusion, J. Math. Biol., 7 (1979), pp. 231-241.

[4] P. C. FIFE, Mathematical Aspects of Reacting and Diffusing Systems, Lecture Notes in Biomathematics 28, Springer-Verlag, New York, 1979.

[5] A. C. FOWLER, Approximate solution of a model of biological immune responses incorporating delay, J. Math. Biol., 13 (1981), pp. 23-45.

[6] J. K. HAle, Theory of Functional Differential Equations, Springer-Verlag, New York, 1977.

[7] G. R. IVANitsky, V. I. KRINSKy, A. N. ZAikin AND A. M. Zhabotinsky, Autowave processes and their role in disturbing the excitability of distributed excitable systems, Soviet Sci. Rev. D (Biology Review), 2 (1981), pp. 279-324.

[8] G. S. JONES, On the nonlinear differential difference equation $f^{\prime}(x)=-\alpha f(x-1)\{1+f(x)\}$, J. Math. Anal. Appl., 4 (1962), pp. 440-469.

[9] J. P. KEENER, Waves in excitable media, this Journal, 39 (1980), pp. 528-548.

[10] A. N. Kolmogorov, I. G. Petrovsky and N. S. Piskunov, A study of the equation of diffusion with increase in the quantity of matter and its application to a biological problem, Bjul. Moskovskogo Gos. Univ., 1 (1937), pp. 1-26.

[11] Y. Kuramoto, Diffusion induced chaos in reaction systems, Suppl. Prog. Theor. Phys., 64 (1978), pp. 346-367.

[12] J. LIN AND P. B. KAHN, Phase and amplitude instability in delay-diffusion population models, J. Math. Biol., 13 (1982), pp. 383-393.

[13] N. Macdonald, Time Lags in Biological Models, Lecture Notes in Biomathematics 27, SpringerVerlag, New York, 1978.

[14] K. MAGINU, Stability of spatially homogeneous periodic solutions of reaction-diffusion equations, J. Differential Equations, 31 (1979), pp. 130-138.

[15] R. M. MAY, Stability and Complexity in Model Ecosystems, Princeton Univ. Press, Princeton, NJ, 1973.

[16] J. Maynard-Smith, Models in Ecology, Cambridge Univ. Press, Cambridge, 1974.

[17] A. OKubo, Diffusion and Ecological Problems: Mathematical Models, Biomathematics 10, SpringerVerlag, New York, 1980.

[18] F. Rothe, Asymptotic behavior of the solutions of the Fisher equation, Biological Growth and Spread, W. Jäger, H. Rost and P. Tautu, eds., Lecture Notes in Biomathematics, 38, Springer-Verlag, New York, 1980, pp. 279-289.

[19] A. SCHIAfFINO AND A. TESEI, Time periodic solutions for Volterra population equations, presented at the III Simposio Italiano di Dinamica della Popolazioni, Pallanza, Italy, September, 6-8, 1979.

[20] V. A. Vasilev, Yu. M. Romanovskil And V. G. Yakhno, Autowave processes in distributed kinetic systems, Sov. Phys. Usp., 22 (1979), pp. 615-639.

[21] M. G. Velarde ANd L. L. Bonilla, Competition between time delay and diffusion in a model ecological problem, Symmetries and Broken Symmetries in Condensed Matter Physics, N. Boccara, ed., IDSET, Paris, France, 1981, pp. 391-398.

[22] - The spruce budworm-forest and other ecosystems, presented at the Colloque sur les Rhythmes en Biologie, Chimie, Physique et autres champs d'application, Journees S.M.F., Marseille, September, 1981, Springer, New York, to appear.

[23] W. C. Clark, D. D. Jones And C. S. Holling, Patches, movements, and population dynamics in ecological systems: a terrestrial perspective, in Spatial Patterns in Plankton Communities, J. H. Steele, ed., Plenum Press, New York, 1978, pp. 385-432.

[24] J. J. TYSON AND P. C. FIFE, Target patterns in a realistic model of the Belousov-Zhabotinskii reaction, J. Chem. Phys., 73 (1980), pp. 2224-2237.

[25] K. UCHIYAMA, The behavior of solutions of some nonlinear diffusion equations for large time, J. Math. Kyoto Univ., 18 (1978), pp. 453-508. 
[26] S. N. Chow, J. Mallet-Paret And J. A. Yorke, Global Hopf bifurcation from a multiple eigenvalue, Nonlinear Anal., 2 (1980), pp. 753-763.

[27] S. HASTINGS, J. TYSON AND D. WEBSTER, Existence of periodic solutions for negative feedback cellular control systems, J. Differential Equations, 25 (1977), pp. 39-64.

[28] H. KIELHÖFER, Degenerate bifurcation at simple eigenvalues and stability of bifurcating solutions, J. Funct. Anal., 38 (1980), pp. 416-447. 